\title{
A Structured Framework to Assess the Business Application Landscape of Blockchain Technologies
}

\author{
Gianluca Salviotti \\ SDA Bocconi School of \\ Management - DEVO Lab \\ gianluca.salviotti@sdabocconi.it
}

\author{
Leonardo Maria De Rossi \\ SDA Bocconi School of \\ Management - DEVO Lab \\ $\underline{\text { leonardo.derossi@sdabocconi.it }}$
}

\author{
Nico Abbatemarco \\ SDA Bocconi School of \\ Management - DEVO Lab \\ nico.abbatemarco@sdabocconi.it
}

\begin{abstract}
Blockchain is emerging as a game changing technology in many industries. Although it is increasingly capturing the business community's attention, a comprehensive overview of commercially available applications is lacking to date.

This paper aims to fill this gap. Firstly, we propose a structured approach to assess the application landscape of blockchain technologies. To build our framework, we relied on largely accepted classifications of blockchains, based on protocols, consensus mechanisms and ownership, as well as on the most cited application areas emerging from the literature.

Secondly, we applied the framework on a database of 460 released blockchains. The analysis confirms a dominance of applications for cryptocurrencies, financial transactions and certification purposes, with a prevalence of permissionless platforms. We also found new application fields that go far beyond the seven initial areas addressed by the current body of knowledge, leading to some interesting takeaways for both practitioners and IS researchers.
\end{abstract}

\section{Background}

A blockchain is a form of network-distributed ledger, whose users play an active role in keeping it constantly updated. The first concept of a blockchain was designed in 2008 and implemented in 2009 as the core protocol of the digital currency Bitcoin. This first blockchain was conceived with the purpose of allowing peer-to-peer transactions through Bitcoin, and it has since been a source of inspiration for thousands of different developers.

\footnotetext{
1 According to the CryptoCurrency Market Capitalization data provided by Coin-Market Cap https://coinmarketcap.com/
}

The term "blockchain 2.0" is often used to refer to all the most recent applications of the blockchain infrastructure, whose potential uses go far beyond exchanging value without an intermediary [1]. Its benefits might include advanced security [2], data transparency [3], digital intelligence [4], disintermediation and many others [5].

Based on these benefits, according to a recent report from PwC, "blockchain could become a force anywhere trading occurs, trust is at a premium, and people need protection from identity theft" [6]. Such a potential pervasiveness is making blockchain one of the most promising technologies in the digital arena, as recognized by leading institutions and market analysts such as the World Economic Forum [7] and Gartner Group [8].

The increasing enthusiasm of the business community around blockchain technologies is also powered by several concurring trends. First, looking to the native application field of blockchain technologies, the global market of cryptocurrencies is continuously growing and has exceeded $\$ 160$ billion on August 2017, starting out at $\$ 10$ billion at the beginning of the year and hitting $\$ 100$ billion in June ${ }^{1}$. While Bitcoin is still the most valuable cryptocurrency by market capitalization, other lesser-known digital currencies are also growing in value, as a proof of the increasing interest in these new currencies and their underlying technical platforms [9]. Second, both the top ICT players and the largest Venture Capitalists are heavily investing in new companies focused on blockchain technologies, applications and standards: according to Friedlmaier et al. [10], the overall investment in blockchain-focused start-ups has reached $\$ 1.5$ billion during 2016. Third, looking to new application fields of the blockchain, several big names far from financial services, such as Walmart and Maersk, have started to launch implementation initiatives aimed at testing the benefits of distributed ledger technologies [11]. Lastly, 
several companies, research institutions and industry consortia are joining forces to create blockchain standards, platforms and applications. Examples of broad networking initiatives aimed at advancing blockchain technologies for either cross-industry applications or industry specific applications are, respectively, Hyperledger and R3.

Unfortunately, the combined effect of these trends is leading to a hype effect around blockchain [12] [13] [14]. While it is commonly accepted that blockchain could lead to radical changes in many industries [10] [15], with a potential impact on the whole economy [1] [16], several authors focus on the medium-to-long time needed in order to actually experience some transformational impacts of this technology. This is mainly due to the foundational nature of blockchain, as highlighted by Iansiti and Lakhani [14]: "It has the potential to create new foundations for our economic and social systems. But while the impact will be enormous, it will take decades for blockchain to seep into our economic and social infrastructure. [...] Many barriers-technological, governance, organizational, and even societal -will have to fall".

Consistently, most of the efforts spent by the academic world in the last 5 years have been devoted to solve the challenges that are slowing down the potential disruption led by blockchain and distributed ledger technologies, with a main focus on Bitcoin and other cryptocurrency applications [17]. Very few works have pointed their attention on alternative applications of blockchain technologies [10].

As a consequence, business leaders and practitioners are relying on newly founded, non-academic organizations such as, for example, the Blockchain Research Institute ${ }^{2}$ or the Institute for Blockchain Studies $^{3}$, to address the following unanswered questions:

- Which are the main business applications of blockchain, other than cryptocurrencies?

- Which are the most affected industries?

- Which are the main technical features of blockchain platforms currently implemented?

- Who owns the blockchains in current business implementations?

Moreover, the business application landscape of blockchain technologies has been analysed in a fragmented and heterogeneous way, as shown by Notheisen et al. [13] . According to these authors, the IS Research could play a leading role in facilitating the transition from the hype phase to cross-industry market applications of blockchain technologies. However, in

\footnotetext{
${ }^{2}$ The Blockchain Research Institute, based in Toronto, is a private research institution that aims at exploring blockchain use cases, opportunities and implementation challenges.
}

order to make a valuable contribution to the business community, scholars should leverage on a common language and approach to structure their research effort.

Following this research path, this paper aims to propose a possible approach to study and assess the business application landscape of blockchain.

In the first part of the paper (Par. 2,3,4), starting from the key building blocks addressed by the literature, we present the most relevant technical and functioning features which characterize this technology. These features are the starting point to classify blockchain platforms and will be included into our framework to analyse the current application landscape.

In the second part of the paper (Par. 5) we focus on the seven most relevant applications of blockchain that have been addressed by the literature. In our opinion, the business application landscape of blockchain technologies is much broader and entails, with different intensity, several industries. This is why, in the third part of the paper (Par. 6), we apply the framework on 460 companies operating in the blockchain market, selected from public available data sources.

The last part of the paper (Par. 7) describes our main findings after the first application of the framework, with some remarks for business executives and researchers interested in blockchain implementations.

\section{The Blockchain technical pillars}

\subsection{Blockchain - Technological overview}

It is largely accepted that blockchain is based on three main pillars, related to the concepts of decentralization, cryptography and consensus [10]:

- Distributed computation

- Public key cryptography

- Decentralized consensus

An effective interlacement of the first two pillars has been researched since the late $1980 \mathrm{~s}$, in various attempts to create a virtual monetary ecosystem, the most important of which has been represented by D. Chaum's Digicash [18] in 1990. On the other side, decentralized consensus was initially deployed as a DDoS countermeasure in Adam Back's Hash-cash [19] in 1997. Finally, it was the close-knit intertwinement of the three pillars that gave birth to the blockchain mechanism we know today.

This combination has been firstly examined in the theoretical works of Wei Dai [20] and Nick Szabo [21], and later deepened in the first blockchain paper ever published, S. Nakamoto's [22] Bitcoin White Paper.

\footnotetext{
${ }^{3}$ The Institute for Blockchain Studies is an independent non-profit research institute examining the theoretical, philosophical, and societal implications of blockchain technology.
} 


\subsection{Pillar 1: Distributed Computation}

Blockchain is - usually - a shared public ledger. In the broadest sense of the term, distributed computing means that the computation power is shared among multiple systems which may also be in different locations [23]. Generally, each active user is required to download a full copy of the blockchain, which includes all the history of that specific protocol until that moment. For example, in the Bitcoin blockchain, each user must download all the transactions ever recorded on the blockchain to enter the network. After this step, each node can run independently, processing any incoming transaction and propagating it further: the stored transactions are automatically synchronized among all the nodes - thus, there is no need of a central node processing and distributing data. Moreover, each node can contribute to reach the consensus. This model of computation could be extended to many other services, such as Domain Name Server (DNS) [24].

\subsection{Pillar 2: Public Key Cryptography}

Public-key cryptography, or asymmetric cryptography, is an encryption scheme that employs two mathematically related numbers - a first one called private key, and, derived from it through a complex mathematical function, a second one called public keyeach one performing a unique function [25].

The public key is used to encrypt, while the private key is used to decrypt: together they represent the digital signature of a user. It is computationally unfeasible to calculate the private key starting from the public key [26]. Therefore, public keys can be freely shared, providing users with an easy and convenient method for encrypting content and verifying digital signatures, while at the same time private keys can be kept secret, ensuring that only their owners can decrypt content and create digital signatures [27] [28].

\subsection{Pillar 3: Decentralized consensus}

As mentioned above, blockchain is basically a network-distributed database whose nodes continuously record information in "blocks", assembled in a unique "chain". To achieve decentralized consensus means that one party must no longer go through a central authority, or trust the other party, to share information (including information in the form of value transactions).

\footnotetext{
${ }^{4}$ Less used distributed consensus mechanisms such as PoET, PoC, PoI, FBA, have been considered in our field analysis and included into the framework, but they are not described in this paragraph.
}

Many consensus mechanisms have been developed in these years. However, given the non-technical nature of this work, the detailed discussion of distributed consensus mechanisms is limited to the most common use cases, i.e. Practical Byzantine Fault Tolerance (PBFT), Proof of Work (PoW) and Proof of Stake (PoS). Some slightly modified versions of these consensus mechanisms, such as distributed $\mathrm{PoS}(\mathrm{dPoS})$ or delayed PoW (dPoW), will be considered as assimilated to their original versions for the purpose of this work ${ }^{4}$.

2.3.1. Practical Byzantine Fault Tolerance (PBFT). The PBFT algorithm works as follows. Each node owns a public key. When a node receives a message, it uses the message in conjunction with its public key to run a computation or operation. This computation in turn allows that individual node to express its opinion on the message in question. Then, after reaching its individual decision, the node shares that decision with all the other nodes in the system. Consensus is reached on the basis of the total decisions submitted by all nodes [29]. Since they always engage all nodes of the network for each and every transaction, PBFT and other state-machine replication protocols are often challenged for their scalability in terms of number of nodes (replicas) [30]. An example of blockchain that relies on the PBFT for consensus is Hyperledger.

2.3.2. Proof-of-Work algorithm (PoW). It's the most well-known method for reaching consensus on a blockchain and is the one deployed by Bitcoin. In contrast to the solution used in PBFT, PoW does not require all the nodes on a network to submit their individual conclusions to reach a consensus. Instead, PoW uses a hash function ${ }^{5}$ of fixed size to create conditions, under which a single participant is permitted to announce their conclusions about the submitted information, and those conclusions can then be independently verified by all the other system participants. The key reason why the hash function is used is its irreversibility: a hash function cannot be reverse-engineered. In fact, false conclusions are prevented by the parameters of the hash function, ensuring that false information will fail to compute in an acceptable way.

As a result, producing a proof of work becomes a random process with low probability of success, so that a lot of trial and error is required on average before a valid proof of work is generated. This means every user is sure, and can freely verify, that a certain amount of work has been spent to create a new block; for a malicious entity to change the state of the ledger it will

\footnotetext{
${ }^{5}$ A hash function transforms a string of characters into a shorter fixedlength value that represents the original string, and is used to index and retrieve items in a database.
} 
require having more computational power than the entire existing network

2.3.2. Proof-of-Stake algorithm (PoS). The key reason for choosing a proof of stake mechanism is that mining is done by all the stakeholders in the ecosystem who have a financial interest in the chain [23]. PoS replaces the hash function calculation with a digital signature, which proves ownership of the stake. The network (pseudo) randomly selects an individual to approve new messages (to confirm the validity of new information submitted to the database) on the basis of their proportional stake in the network. In other words, instead of any individual attempting to calculate a value in order to be chosen to establish a consensus point, the network itself decides who will announce the results, and system participants are exclusively and automatically entered into that lottery in direct proportion to their total stake in the network. Three examples of blockchain providers using this consensus method are Bitshares, Nxt and Blackcoin. Ethereum is currently planning to switch from PoW to PoS with its new update "Casper".

\section{Ownership of the Blockchain}

We can identify two types of blockchain on the basis of the ownership of the platform [31] [32]:

- Permissionless blockchain

- Permissioned blockchain

In a permissionless blockchain anyone over the Internet can read, send transactions and participate in the consensus process. Thus, anyone is free to be an active part of the entire network. Permissionless blockchains are always open source - which means that the underlying algorithm is completely public. Moreover, a previous relationship with the ledger is not required. A permissionless blockchain is mostly appropriate when a network needs to be decentralized. It is also suitable to ensure full transparency of the ledger or higher level of anonymity ${ }^{6}$. Costs are higher and speed is slower than on a private chain, but it is still a faster and less expensive method than the ones used today. The two most relevant examples of permissionless blockchains are Bitcoin and Ethereum. For this type of blockchain, the most appropriate consensus algorithms are PoW and PoS.

On the other side, a permissioned blockchain is kept centralized to one - or more - authorized user. In this case, the authorized user(s) verifies each transaction. Read permissions may be public or restricted to an

\footnotetext{
${ }^{6}$ In a permissionless blockchain we can register different grade of anonymity widening from full-anonymity (e.g. Monero) to pseudoanonymity (e.g. Bitcoin).
}

arbitrary extent. Likely applications include database management, auditing, and more, that are internal to a single company. This type of blockchain enables greater efficiency and allows transactions to take place much faster. Two significant examples are Hyperledger and Ripple. For this type of blockchain, the most appropriate consensus algorithm is PBFT.

\section{Smart Contracts}

Smart contracts are increasingly becoming a cornerstone for enterprise blockchain applications and will likely become a pillar of blockchain technology [1].

Basically, a smart contract is a computer program code that is capable of facilitating, executing, and enforcing the negotiation or performance of an agreement (i.e. contract) using blockchain technology. Vitalik Buterin ${ }^{7}$ described the smart contracts as "contracts that can be used to encode arbitrary state transition functions, as well as many others that we have not yet imagined, simply by writing up the logic in a few lines of code" [33].

These contracts act as an agreement, where the terms of the agreement can be pre-programmed within a blockchain infrastructure with the ability to selfexecute. The main goal of a smart contract is to enable two anonymous parties to trade and do business with each other, usually over the internet, without the need for a middleman.

Even if there are significant concerns that smart contracts will encounter considerable difficulty adapting to current legal frameworks regulating contracts across jurisdictions [34], the potential applications basing on this "technological tool" are infinite.

There are countless practical use cases developed, where blockchain technology is being applied to achieve significant benefits, and smart contracts are used in most of these applications [35].

The most important blockchain platform for the deployment of smart contracts is commonly considered Ethereum. Even if Ethereum is the most-known blockchain for smart contract, there are other platforms which allow user to develop their own smart contracts, such as Stratis, Lisk, Nem, Nxt.

\section{Blockchain applications}

Companies across many industries have already started to adopt blockchain technologies [36]. Both the business and technical literatures are providing use

\footnotetext{
${ }^{7}$ Vitalik Buterin is Ethereum co-founder and it is widely considered one of the most relevant programmer among the entire blockchain community.
} 
cases where blockchain technologies are applied to enhance legacy systems, as well as to offer new services. In the following sub-paragraphs, we provide a summary of the most prominent and recurring applications already identified by the relevant literature [16] [36] [37], with the aim to test and verify them with our framework.

\subsection{Advanced Tracking}

Blockchain is the missing link to settle scalability, privacy and reliability concerns in the Internet of Things [38]. The current IoT ecosystems rely on centralized, brokered communication models, otherwise known as the server/client paradigm. All devices are identified, authenticated and connected through cloud servers that sport huge processing and storage capacities [39].

Blockchain could be used in tracking billions of connected devices, enabling the processing of transactions and coordination between devices [40]. The decentralized consensus will create a more resilient ecosystem for devices to run on, practically eliminating a single point of failure. Moreover, the cryptographic algorithms can guarantee a high level of privacy for the users [41].

Adopting a standardized peer-to-peer communication model to process the hundreds of billions of transactions between devices will significantly reduce the costs associated with installing and maintaining large centralized data centres and will distribute computation and storage needs across the billions of devices that form IoT networks. This will prevent failure in a single node to bring the entire network to a halting collapse [42].

The whole supply chain could benefit from the adoption of tracking systems enabled by both IoT and blockchain technologies [43]. As mentioned by Zhao et al. [44], Walmart plans to use technology developed by the Hyperledger Project, an open source software project that builds blockchain tools developed by IBM and the Linux Foundation.

\subsection{Certification - Antifraud applications}

Blockchain represents one of the best ways to fight various types of fraud - such as subsidized housing sales and mileage manipulation in second-hand vehicles. In a blockchain it is (almost) impossible to rewrite any data already registered. Thus, it is the perfect tool to develop anti-fraud registries capable of putting an end to fraud schemes such as the ones mentioned above [45]. Timestamping data in an unalterable state while maintaining confidentiality is a perfect solution to avoid fraud actions. It allows anyone to store a hash of any document into a blockchain, thus proving it existed at the time when a particular block was created [46].

One of the most relevant example of this services is Everledger. Everledger built a global, digital ledger that tracks and protects valuable assets throughout their lifetime journey.

\subsection{Cloud storage}

Scholars and practitioners suggested alternative application of blockchain while dealing with the limits of Bitcoin. Miller et al. [47] suggest a modification to Bitcoin that repurposes its mining resources to achieve a more broadly useful goal, i.e. distributed cloud storage of archival data. The principle is that on a blockchain platform, the same users can host their surplus storage capacity and renters can purchase this extra-storage and upload files. Basically, the blockchain could enable [48]:

- A complete decentralization and a real redundancy basically eliminating the possibility of one-point-offailure;

- A high privacy and security level considering that no node controls user data nor has a direct access to user files, but nodes only stores encrypted fragments of user data;

- A significant cost reduction. For instance, blockchain storage costs around $2 \$$ per terabyte per month, compared with Amazon S3's 25\$ per terabyte per month.

One of the most studied, commercially available service offering storage trough blockchain is the one provided by Storj. Storj is based on an open source software project that brings blockchain technology to assure files are both secure and not easily viewed or shown to unauthorized users [49]. This system enables users to store data in a secure and decentralized manner. It does this through the use of blockchain features such as a transaction ledger, public/private key encryption, and cryptographic hash functions.

\subsection{Cryptocurrencies}

Cryptocurrencies are any kind of electronic money created using cryptographic technology. They regulate their own issuing and ensure the legitimacy of transactions conducted through them. They can be considered as the original and first-proven application of blockchain technologies.

Cryptocurrencies are open-source algorithms, which can (usually) be programmed by anyone and facilitate peer-to-peer financial networking without the need for third party arbitration, thereby reducing the dependency on banking systems. This creates an open environment, 
which has tremendous economic potential in an increasingly digitalised and globalised world. However, as showed by Syed et al. [50] cryptocurrencies have some weaknesses, such as digital security, market regulation and speculative attacks among others. Most of the cryptocurrency market is shared between Bitcoin and Ethereum.

\subsection{Digital Identities}

Currently, a general consumer is forced to submit his document - ID scan, copy of passport, photo of credit card - to third party services over the Internet for verification purposes. These documents are stored on centralized servers and they become an easy quarry for hackers or hoodlums. Identity theft is a humongous problem of these last years [51].

Identity verification through blockchain technology could be the solution to this problem, allowing consumers to verify their identity while there is no centralized storage of identity documents involved [52]. The main benefits for consumers of a blockchain-based digital ID are:

- General Trust: with customers in control of their identity data and a framework for rapid verification, blockchain creates an environment more conducive to mutual trust;

- Efficiency: customers bear the brunt of inefficiencies, wasting time filling out forms, repeating conversations and gathering documentation;

- Security: with users directly controlling their ID and every action is recorded in an immutable ledger, it is less likely to find problems with ID management, theft, security and inconsistency;

- Privacy and advanced data sovereignty: users could be the only owners of their personal data.

One of the most significant examples of companies developing Digital ID is Shocard, which is built on a public blockchain.

\subsection{Energy Management and Distribution}

Blockchain could be used to develop a peer-to-peer energy market, which can guarantee that operational constraints are respected and payments are fairly rendered, without relying on a centralized utility company or micro-grid aggregator. In other words, blockchain could be used to develop a digital contract permitting an individual party to conduct and bill a transaction (e.g. a sale of electricity) directly with another party (peer-to-peer). Aitzhan et al. [53] implemented a proof-of-concept for decentralized energy trading systems using blockchain technology, multi-signatures, and anonymous encrypted messaging streams, enabling peers to anonymously negotiate energy prices and securely perform trading transactions. Hukkinen et al. [54] have analysed an Ethereum-based application of smart contracts to facilitate market matching between individual producers and consumers of electricity.

We can also mention the micro-grid project in Brooklyn, where residents with solar panels can sell excess energy directly to their neighbours in a peer-topeer transaction, which leverages blockchain technology [55].

Also, energy generation from renewable sources is getting attention as a field that could benefit from blockchain technologies [56].

SolarCoin is an example a new environmentally friendly currency backed by the solar output of photovoltaic solar panels. Participants in the network get SolarCoins by submitting a proof of solar electricity generation in the form of a verifiable meter reading.

\subsection{Financial Transactions}

Blockchain technologies can potentially allow the entire financial services industry to dramatically optimize business processes thanks to a new secure, transparent and efficient system of data sharing [57]. The existing capital markets infrastructure is slow, expensive, and often requires several intermediaries [58]. The main benefits for the financial services would be: instant settlements, improved capital optimisation, reduced counterparty risk improved contractual performance, increased transparency and reduced error handling and reconciliation. A significant application in financial transactions could be that of remittances. Western Union, MoneyGram, and all the companies operating in this field move about $\$ 550$ billion through their networks, according to the World Bank, and there is probably about $\$ 150$ billion to $\$ 200$ billion that is unreported [59]. The average fee is $10 \%$, maybe a little bit higher when considering all the shadow transactions. Potentially, up to $\$ 63$ billion could be saved by using a blockchain-based remittances system rather than the traditional systems, one that could drive the fees down to $1 \%$. The output of a transaction executed through a typical remittances company is transferred between seven to nine intermediaries on average before reaching its recipient. Underbanked individuals who do not have access to conventional banking system pay the highest fees of this system, which is unfortunate because they are also the people who could use the money the most.

Another relevant application of blockchain in the financial sector could be in P2P transactions. Abra is one of the most relevant company using blockchain technologies for both $\mathrm{P} 2 \mathrm{P}$ payments and remittances 
Finally, blockchain could have a relevant role in crowdfunding services. There have been a few experimental attempts to build decentralized crowdfunding platforms on the blockchain. Other important applications could be developed to deal with cross-border payments, derivatives, foreign exchange markets, post trade processing settlements.

\section{Application landscape assessment}

\subsection{Assessment framework and Methodology}

On the basis of the previous analysis and the main academic/technical literature, we built an assessment framework to map blockchain applications according to Table 1. As shown in the Table, the framework entails 5 main building block variables that could lead to a better understanding of the features that qualify a blockchain solution: industry, consensus mechanism, ownership, type of application. A field "application description" has been added in order to collect additional information about the solution.

In order to perform an analysis as coherent and complete as possible, we built a dataset gathering data from two different sources, for a total of 460 observations. The main is CoinMarketCap [60]. As of July $17^{\text {th }}$ 2017, this database contained 979 entries, divided into the currencies and assets categories.

Table 1. Assessment Framework

\begin{tabular}{|c|c|c|}
\hline Variable & Description & Reference \\
\hline Industry & $\begin{array}{l}\text { The industrial sector affected by } \\
\text { the blockchain application }\end{array}$ & {$[61]$} \\
\hline $\begin{array}{l}\text { Consensus } \\
\text { mechanism }\end{array}$ & $\begin{array}{l}\text { The consensus mechanism at the } \\
\text { base of the considered protocol }\end{array}$ & $\begin{array}{l}{[29][62]} \\
{[63][64]}\end{array}$ \\
\hline Ownership & $\begin{array}{l}\text { Distinction between } \\
\text { permissionless and permissioned } \\
\text { blockchains }\end{array}$ & $\begin{array}{l}{[3][31]} \\
{[32][65]}\end{array}$ \\
\hline $\begin{array}{l}\text { Blockchain } \\
\text { Protocol }\end{array}$ & $\begin{array}{l}\text { Blockchain protocol on which the } \\
\text { application is based }\end{array}$ & $\begin{array}{c}{[3][23]} \\
{[36][62]} \\
{[66]} \\
\end{array}$ \\
\hline $\begin{array}{l}\text { Type of } \\
\text { application }\end{array}$ & $\begin{array}{l}\text { The purpose of application } \\
\text { performed. The categories in } \\
\text { which this item was subdivided } \\
\text { derive from the } 7 \text { main areas } \\
\text { summarized in Par. 5, plus others } \\
\text { emerging from the empirical } \\
\text { analysis }\end{array}$ & $\begin{array}{c}\text { [1] [4] [16] } \\
\begin{array}{c}\text { From [36] } \\
\text { to [59] }\end{array} \\
\text { [66] [67] }\end{array}$ \\
\hline $\begin{array}{l}\text { Application } \\
\text { description }\end{array}$ & $\begin{array}{l}\text { A brief description of what the } \\
\text { blockchain does/aims to achieve }\end{array}$ & $\begin{array}{c}\text { Blockchain } \\
\text { websites }\end{array}$ \\
\hline
\end{tabular}

To maintain a good compromise between the completeness and the significance of the sample, we excluded the protocols with a market capitalization inferior to $500,000 \$$ at that date, obtaining 401 valid entries. We furthermore integrated the sample with other 59 observations, derived from the Crunchbase portal using, as query for the research, the word "blockchain". All the blockchains thus obtained have been subsequently analysed and qualified according to the framework. It should also be noted that, for the purpose of the analysis:

- purely theoretical/conceptual blockchains have not been considered;

- projects related with the blockchain world, but that do not make use of a blockchain protocols in order to work (for example blockchain consulting or pure mining companies), have not been considered.

\subsection{Application of the framework: results}

First of all, as shown in Table 2, it can be noticed that, thanks to analysis, the initial list of 7 applications (Par. 5) has been significantly broadened. While Cryptocurrency, Financial Transactions, Certification, Digital Identity confirm their relative relevance, Energy distribution and Advanced tracking have been overtaken (at least in terms of number of released blockchains) by other application fields such as Platform Development, Gaming, P2P Content Distribution and Digital Voting \& Government.

If we add to this picture a breakdown from the ownership perspective, we can provide some interesting evidences. The distribution by application of permissioned (Permd) and permissionless (Perms) blockchains respects the proportion between the two categories (1:8). Notably, only some types of application break this rule: Cryptocurrency, Digital Voting \& Governance and P2P Content Distribution mostly belong to the public ecosystem, while at the permissioned level there seems to be a focus on Digital ID, Digital Rights Management, Financial Transaction, Platform and Tracking \& Control. In particular, Tracking \& Control is the only application more commonly found among permissioned blockchains.

Table 2. Type of application for blockchains

\begin{tabular}{|l|r|r|r|}
\hline Overall & \multicolumn{1}{|c|}{ Permd } & \multicolumn{1}{c|}{ Perms } & \multicolumn{1}{l|}{ Total } \\
\hline ADV \& Customer Loyalty & 1 & 9 & 10 \\
\hline Certification & 7 & 47 & 54 \\
\hline Cloud Storage & & 4 & 4 \\
\hline Cryptocurrency & 1 & 121 & 122 \\
\hline Cybersecurity & 1 & 7 & 8 \\
\hline Digital Identity & 4 & 16 & 20 \\
\hline Digital Rights Management & 2 & 5 & 7 \\
\hline Digital Voting \& Government & 1 & 14 & 15 \\
\hline Energy Distribution & & 2 & 2 \\
\hline Financial Transaction & 26 & 71 & 97 \\
\hline Gaming & 1 & 22 & 22 \\
\hline P2P Content Distribution & 14 & 45 & 16 \\
\hline Platform Development & & 4 & 59 \\
\hline Prediction Market & & 9 & 9 \\
\hline Smart Contracts & 9 & 2 & 11 \\
\hline Advanced Tracking & $\mathbf{6 7}$ & $\mathbf{3 9 3}$ & $\mathbf{4 6 0}$ \\
\hline Total
\end{tabular}


Figure 1 illustrates the use frequency of the consensus mechanisms.

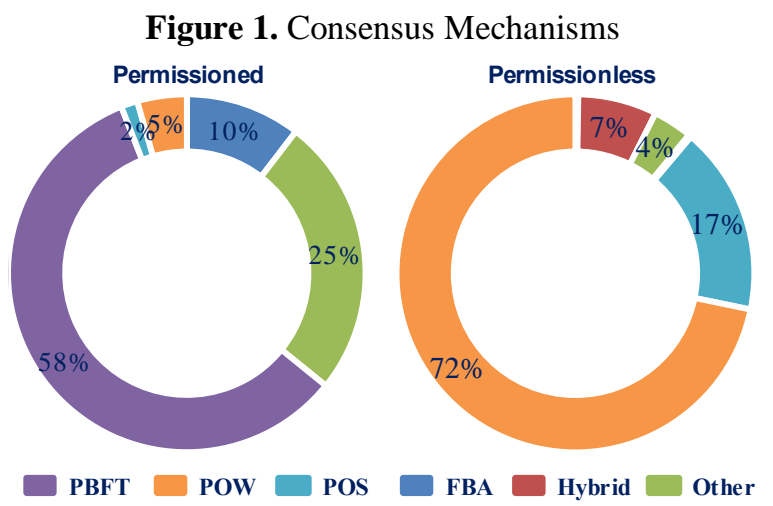

The $96 \%$ of the permissionless cluster is shared between PoW (72\% of all the observations), PoS (17\%), and a hybrid PoW/PoS model (7\%). The PoW mechanism plays an important role also in the permissioned cluster (5\% of all the observations); here, however, it cedes the primacy to PBFT (58\%). The high frequency of the "Other" category in the permissioned cluster is due to a high rate of confidentiality: many providers refused to publicly state what kind of consensus mechanism they are currently using.

Figure 2. Distribution by industry

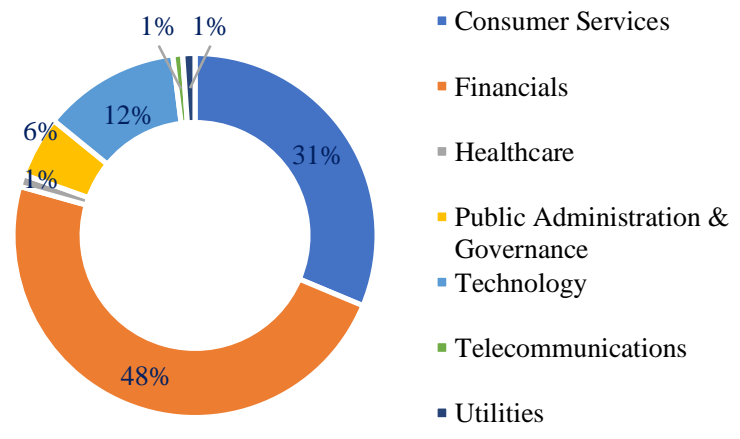

Figure 2 takes into account the representativeness of the industries, in which blockchain has been used.

As expected, Financial Services is the most represented (48\%), followed by Consumer Services $(31 \%)$. In total, these two industries account for over three-quarters of the market. There are no significant differences between the public and private clusters, which show very similar trends.

Finally, an analysis of the distribution of the market capitalization ${ }^{8}$ by type of application was performed.
As we can see in Table 3, the prevailing application types remain, albeit at much higher percentages, Cryptocurrency. Platform Development plays an interesting role in terms of market capitalization, since it entails Ethereum and other released blockchains that are used to build tier-two decentralized applications, or DAPPs. The attention focus on using blockchain platforms to build DAPPs, relying on permissionless ownership, is confirmed to be very high.

Table 3. Market capitalization by application

\begin{tabular}{|l|r|r|}
\hline Type of Application (Overall) & $\begin{array}{r}\text { MKT Cap } \\
(\boldsymbol{x} \mathbf{I K} \$)\end{array}$ & $\begin{array}{r}\text { MKT Cap \% } \\
\text { on total }\end{array}$ \\
\hline ADV \& Customer Loyalty & $\$ 103.671$ & $0,13 \%$ \\
\hline Certification & $\$ 970.956$ & $1,23 \%$ \\
\hline Cloud Storage & $\$ 375.771$ & $0,48 \%$ \\
\hline Cryptocurrency & $\$ 49.117 .607$ & $62,41 \%$ \\
\hline Cybersecurity & $\$ 11.723$ & $0,01 \%$ \\
\hline Digital Identity & $\$ 39.911$ & $0,05 \%$ \\
\hline Digital Rights Management & $\$ 9.518$ & $0,01 \%$ \\
\hline Digital Voting \& Government & $\$ 74.685$ & $0,09 \%$ \\
\hline Energy Distribution & $\$ 4.579$ & $0,01 \%$ \\
\hline Financial Transaction & $\$ 1.928 .532$ & $2,45 \%$ \\
\hline Gaming & $\$ 122.535$ & $0,16 \%$ \\
\hline P2P Content Distribution & $\$ 232.064$ & $0,29 \%$ \\
\hline Platform Development & $\$ 25.319 .563$ & $32,17 \%$ \\
\hline Prediction Market & $\$ 389.444$ & $0,49 \%$ \\
\hline Advanced Tracking & $\$ 2.005$ & $0,00 \%$ \\
\hline
\end{tabular}

\section{Conclusion}

The analysis led us to interesting conclusions. First of all, we identified several additional types of application compared to the ones highlighted by the current body of knowledge. Moreover, data confirmed how the most common blockchain application is, without any doubt, Cryptocurrency. The rest of the podium is contended, both in the permissioned and permissionless clusters, between Financial Transaction, Certification, and Platform Development. It is therefore no accident that the same results are mirrored when we point to the reference industries: most of the Financial Transaction applications are related to the Financial industry sector, most of the Certification applications are related to the Consumer Services industry, and Platforms account for almost all the Technology industry cluster. The nature of trusted database of the blockchain makes it ideal for these types of functionalities.

The distinction between permissionless and permissioned becomes more obvious considering the remaining applications. Some of these make sense only in a public environment, as the results testify: Crowdfunding, Cryptocurrency, and P2P Content Distribution. Cloud storage is not present in the

\footnotetext{
${ }^{8}$ Data refers to market capitalization at July 17 th, 2017. Only publicly
} listed companies have been considered. 
permissioned cluster probably due to the presence of different, more diffused technologic solutions in the private sector. It must be underlined how permissioned Tracking \& Control solutions are more numerous than their public counterparts. This is due to Supply Chain being a sector where a certain confidentiality could be required.

The analysis furthermore highlights the current dominance of Ethereum for the development of DAPPs, especially at the public level, but with a non-indifferent impact even at the private level.

The state-of-the-art of consensus mechanisms is also clear. The PoW remains the most widely distributed consensus mechanism, with a clear prevalence over others. This is mainly due to its being Bitcoin's consensus mechanism: as long as the Bitcoin system does not show blackouts, the consensus mechanism at its base is almost automatically considered the most stable of all, taking into account the high number of hackers that attacks the network every day.

However, it must be noted that innovative solutions are being progressively tested.

Our study provides both practitioners and IS Researchers with useful insights for their goals.

From a managerial point of view, the analysis clearly shows which industries are the most impacted by blockchain technologies: managers in the most affected industries should no longer postpone the launch of blockchain-inspired initiatives, being those initiatives either large implementations or pilot tests aimed at growing the maturity level on this technology. Once decided to launch a project, other key options are related to what type of platform to implement (permissioned vs. permissionless), as well as on which consensus mechanism to rely on. Our study provides a useful stateof-the-art to address these decisions. Permissionless solutions based on PoW seem to represent the status$q u o$, corroborating the importance of the adoption of the infrastructure by a broad and public network of users as a key success factor for blockchain implementations [14] [68]. On the other side, managers working in the less impacted industries might look at blockchain as a unique opportunity for innovation [10], pointing their attention on some cross-industry applications such as, for example, advanced tracking, that is claimed to be a disruptive solution for supply chain management after the Walmart's experience [11].

From a research perspective, the framework proposed in this work could represent a starting point for furtherly investigating the business application landscape of blockchain technologies. First, the framework could be broadened with additional variables, as they will emerge from the literature. Second, it could be applied to a wider sample of blockchains in order to confirm the findings in terms of blockchain applications. Third, it could be adapted and improved in order to derive a decisional framework for practitioners interested in investing in blockchain technologies.

\section{References}

[1] M. Swan, "Blockchain. Blueprint for a new Economy", O'Really Media, 2015.

[2] A. Dorri, et al., "Blockchain for IoT security and privacy: The case study of a smart home," IEEE International Conference, PerCom Workshops, 2017

[3] M. Pilkington, "Blockchain Technology: Principles and Applications", in Research Handbook on Digital Transformations, by F. Olleros, M. Zhegu, Elgar, 2016

[4] M. Swan, "Blockchain Thinking: The Brain as a DAC (Decentralized Autonomous Organization)", in Texas Bitcoin Conference, 2015.

[5] P. Tasca, "Ontology of Blockchain Technologies. Principles of Identification and Classification", SSRN 2977811, 2017.

[6] J. Plansky et al., "A strategist's Guide to Blockchain.", PwC Report, 2016.

[7] O. Cann, "These are the top 10 emerging technologies of 2016", in World Economic Forum, 2016. [Online].

[8] K. Panetta, "Gartner's Top 10 Strategic Technology Trends for 2017", 2016. [Online].

[9] C. Bovaird, "Cryptocurrency's Total Market Cap Has Risen Nearly 800\% This Year", Forbes, 2017.

[10] M. Friedlmaier, A. Tumasjan, I.M. Welpe, "Disrupting Industries With Blockchain: The Industry, Venture Capital Funding, and Regional Distribution of Blockchain Ventures", SSRN 2854756, 2016

[11] R. Hacket, "Why Big Business Is Racing to Build Blockchains", Fortune Magazine, 2017.

[12] M. Morini, "From 'Blockchain Hype' to a Real Business Case for Financial Markets", 2016.

[13] B. Notheisen et al., "Breaking Down The Blockchain Hype. Towards A Blockchain Market Engineering Approach", In Proceedings of the 25th European Conference on Information Systems (ECIS), Guimarães, PT, June, 2017.

[14] M. Iansiti \& K. Lakhani, "The Truth about Blockchain." Harvard Business Review 95, no. 1, 2017.

[15] J. Mattila, "The Blockchain Phenomenon. The Disruptive Potential of Distributed Consensus Architectures", No. 38. The Research Institute of the Finnish Economy, 2016.

[16] D. Tapscott \& A. Tapscott, "Blockchain Revolution: How the Technology Behind Bitcoin Is Changing Money, Business, and the World", Penguin, 2016.

[17] J. Yli-Huumo et al., "Where Is Current Research on Blockchain Technology? A Systematic Review", PloS one 11.10 (2016): e0163477, 2016

[18] D. Chaum, "Untraceable Electronic Cash", Goldwasser S. (eds) Advances in Cryptology - CRYPTO' 88, 1990. 
[19] A. Back, "Hashcash - a denial of service countermeasure", 2002.

[20] W. Dai, "B-Money - an anonymous, distributed electronic cash system", http://www.weidai.com/bmoney.txt, 1998

[21] N. Szabo, "The Bitgold proposal", 2005. [Online].

[22] S. Nakamoto, "Bitcoin: A peer-to-peer electronic cash system.", https://bitcoin.org/bitcoin.pdf, 2008

[23] A. Narayanan, "Bitcoin and cryptocurrency technologies", Princeton University Press, 2016.

[24] P. Kovary et al., "Technical Details, Blockchains", 2016.

[25] R.C. Merkle, "Protocols for public key ecosystem"

[26] W. Stallings, "Cryptography and Network Security: Principles and Practice", $7^{\text {th }}$ Edition, Pearson, 2017.

[27] R.L. Rivest, S. Adi, L. Adleman. "A method for obtaining digital signatures and public-key cryptosystems." Communications of the ACM 21.2, 1978.

[28] IEEE 1363: Standard Specifications for Public-Key Cryptography

[29] C. Hammerschmidt, "Consensus in Blockchain Systems. In Short.", Medium Corporation, 2017. [Online].

[30] M. Vukolić, "The quest for scalable blockchain fabric: Proof-of-work vs. BFT replication.", 2015.

[31] V. Buterin, "On Public and Private Blockchains", 2016.

[32] I. Bashir, "Mastering Blokchain", Pakt, 2017.

[33] V. Buterin, "Ethereum White Paper", 2014, [Online].

[34] M. Giancaspro, "Is a 'smart contract' really a smart idea? Insights from a legal perspective", 2017.

[35] M. Bartoletti \& L. Pompianu, "An empirical analysis of smart contracts: platforms, applications, and design patterns", in WTSC 2017, 2017.

[36] W. Mougayar \& V. Buterin, "The Business Blockchain: Promise, Practice, and Application of the Next Internet Technology", John Wiley \& Sons, 2016.

[37] P. Vigna \& M. Casey, "The Age of Cryptocurrency: How Bitcoin and the Blockchain Are Challenging the Global Economic Order", 2016.

[38] M. Atzori, "Blockchain-Based Architectures for the Internet of Things: A Survey", 2016.

[39] A. Banafa, " A Secure Model of IoT with Blockchain", MIT Technology Review, January 5, 2017

[40] S. Huh et al., "Managing IoT devices using blockchain platform", in Advanced Communication Technology, 2017.

[41] K. Prabhu \& K. Prabhu, "Converging blockchain technology with the internet of things", International Education and Research Journal, Vol 3, No 2, 2017.

[42] A. Bahga \& V. Madisetti, "Blockchain Platform for Industrial Internet of Things", Journal of Software Engineering and Applications, n. 9, 2016.

[43] H. M. Kim, "IoT and Blockchain Aware Ontologies for Supply Chain Provenance", SSRN 2818557, 2016.

[44] J. L. Zhao et al., "Overview of business innovations and research opportunities in blockchain and introduction to the special issue.", Financial Innovation, 2:28, 2016.
[45] Y. Cai D. Zhu, "Fraud detections for online businesses: a perspective from blockchain technology ", Financial Innovation, 2:16, 2016

[46] Everis NEXT, "Blockchain disruptive use cases", 2016.

[47] A. Miller, "Permacoin: Repurposing bitcoin work for data preservation.", IEEE Symposium on Security\&Privacy, 2014.

[48] Z. Herbert, "Why blockchains are the future of cloud storage.", 2017. [Online].

[49] S. Wilkinson et al., "Metadisk a blockchain-based decentralized file storage application. Technical Report", 2014. [Online].

[50] A. M. Syed et al., "Cryptocurrency: Next Level in the Evolution of Money", Asian Journal of Research in Business Economics and Management, 2016.

[51] M. Jakobsson \& S. Myers, "Phishing and countermeasures: understanding the increasing problem of electronic identity theft.", John Wiley \& Sons, 2006.

[52] J.-P. Buntinx, "Future Use Cases for Blockchain Technology: Digital ID Verification", 2015. [Online].

[53] N. Z. Aitzhan, "Security and Privacy in Decentralized Energy Trading through Multi-signatures, Blockchain and Anonymous Messaging Streams.", IEEE, 2016.

[54] T. Hukkinen, "A Blockchain Application in Energy", The Research Institute of the Finnish Economy, 2017.

[55] E. Mengelkamp et al. , "Designing microgrid energy markets: A case study: The Brooklyn Microgrid", in Applied Energy, 2017.

[56] L. P. Johnson et al., "Connecting the Blockchain to the Sun to Save the Planet", SSRN Electronic Journal, 10.2139/ssrn.2702639, 2015.

[57] I. Kaminska, "Blockchain promises back-office ledger revolution", 2015. [Online].

[58] Blockchain Technologies, "Blockchain Applications in Financial Services" [Online].

[59] C. G. Cascarilla et al., "Bitcoin, Blockchain, and the Future of Financial Transactions", 2016.

[60] CoinMarketCap, https://coinmarketcap.com, [Online].

[61] F. Russell, "Industry Classification Benchmark (ICB)"

[62] A. Baliga, "Understanding Blockchain Consensus Models", www.persistent.com, April 2017.

[63] S. Seibold \& G. Samman, "Consensus: Immutable agreement for the Internet of value" KPMG, 2016.

[64] I. Bentov et al., "Cryptocurrencies without Proof of Work", https://bravenewcoin.com, 2016.

[65] "Digital Assets on Public Blockchains", BitFury Group, 2016.

[66] A. Antonopoulos, "Mastering Bitcoin: Unlocking Digital Cryptocurrencies", O'Really Media, 2014.

[67] A. Antonopoulos, "The Internet of Money", Merkle Bloom LLC, 2015.

[68] C. Catalini, "The economics of digital cryptocurrencies and the blockchain", MIT Sloan Research Paper No. 5191-16, 2016. 